\title{
Optic Nerve Crush in Mice to Study Retinal Ganglion Cell Survival and Regeneration
}

Evan G. Cameron ${ }^{1, \#, *}$ Xin $\mathrm{Xia}^{1, \#, *}$, Joana Galvao ${ }^{1}$, Masoumeh Ashouri ${ }^{1}$, Michael S. Kapiloff ${ }^{1,2}$ and Jeffrey L. Goldberg ${ }^{1}$

\begin{abstract}
${ }^{1}$ Department of Ophthalmology, Byers Eye Institute, Mary M. and Sash A. Spencer Center for Vision Research, Stanford University School of Medicine, Palo Alto, California 94034, USA; ${ }^{2}$ Department of Medicine and Stanford Cardiovascular Institute, Stanford University School of Medicine, Palo Alto, California 94034, USA

*For correspondence: ecamer1@stanford.edu; xinxia@stanford.edu

\#Contributed equally to this work
\end{abstract}

[Abstract] In diseases such as glaucoma, the failure of retinal ganglion cell (RGC) neurons to survive or regenerate their optic nerve axons underlies partial and, in some cases, complete vision loss. Optic nerve crush (ONC) serves as a useful model not only of traumatic optic neuropathy but also of glaucomatous injury, as it similarly induces RGC cell death and degeneration. Intravitreal injection of adeno-associated virus serotype 2 (AAV2) has been shown to specifically and efficiently transduce RGCs in vivo and has thus been proposed as an effective means of gene delivery for the treatment of glaucoma. Indeed, we and others routinely use AAV2 to study the mechanisms that promote neuroprotection and axon regeneration in RGCs following ONC. Herein, we describe a step-by-step protocol to assay RGC survival and regeneration in mice following AAV2-mediated transduction and ONC injury including 1) intravitreal injection of AAV2 viral vectors, 2) optic nerve crush, 3) cholera-toxin $B$ (CTB) labeling of regenerating axons, 4) optic nerve clearing, 5) flat mount retina immunostaining, and 6) quantification of RGC survival and regeneration. In addition to providing all the materials and procedural details necessary to execute this protocol, we highlight its advantages over other similar published approaches and include useful tips to ensure its faithful reproduction in any modern laboratory. Keywords: Glaucoma, Retinal ganglion cells (RGCs), Degeneration, Neuroprotection, Regeneration, AAV2

[Background] Glaucoma is the leading cause of irreversible blindness worldwide characterized by the progressive degeneration and loss of retinal ganglion cells (RGCs), the central projecting neurons that form the optic nerve connecting the retina to the brain (Quigley, 2011; Tham et al., 2014). Glaucomatous RGC cell death is thought to be induced, in part, by an increase in intraocular pressure (IOP) and concomitant compression of RGC axons as they exit the retina through the optic nerve head (Quigley, 2011; Chang and Goldberg, 2012). Several models of glaucoma in rodents have been developed to study the cellular and molecular mechanisms that underlie RGC degeneration including optic nerve crush (ONC), intracameral injection of microbeads, and intravitreal injection of silicon oil (Sappington et al., 2010; Tang et al., 2011; Templeton and Geisert, 2012; Ito et al., 2016; Zhang et al., 2019). While both the microbead and silicon injection models recapitulate the increase in IOP and induce progressive 
RGC cell death associated with glaucoma, they are not conducive to study axon regeneration due to variability of the insult and incomplete degeneration of RGC axons. Alternatively, ONC has served as a useful preclinical model to study both neuronal survival and regeneration as it induces significant RGC death with little variability and severs all axons allowing confidence that any fibers found past the site of injury are regenerating, rather than spared. Adeno-associated virus serotype 2 (AAV2) specifically and efficiently transduces RGCs following intravitreal injection, making it the principal means to deliver genes (recombinant DNA, shRNA, etc.) into RGCs (Martin et al., 2002; Nickells et al., 2017). Indeed, we've reported the use of AAV2 to deliver therapeutic peptides and shRNA to promote RGC survival and axon regeneration following ONC injury (Moore et al., 2009; Apara et al., 2017; Galvao et al., 2018; Boczek et al., 2019). Here, we describe a comprehensive protocol detailing the use of AAV2 in combination with ONC as a means to study the mechanisms that promote neuroprotection and regeneration, and to identify and characterize candidate molecules with therapeutic potential for the treatment of glaucoma and other optic neuropathies.

\section{Materials and Reagents}

1. Falcon $3 \mathrm{ml}$ transfer pipette (Corning, catalog number: 357524)

2. 6-inch Cotton Tipped Applicators (WWR, catalog number: 89031-270)

3. $\quad V W R^{\circledR}$ Microcentrifuge Tubes (VWR, catalog number: 87003-294)

4. VWR $^{\circledR}$ Superfrost ${ }^{\circledR}$ Plus Micro Slide (VWR, catalog number: 48311-703)

5. Polycin ${ }^{\circledR}$ Ophthalmic Ointment, USP (Perrigo, catalog number: NDC 0574-4021-35)

6. Absorbent Bench Underpads (VWR, catalog number: 82020-845)

7. Kimwipes (Fisher Scientific, catalog number: 06-666)

8. Falcon ${ }^{\circledR} 48$-well clear flat bottom plate (Corning, catalog number: 353078 )

9. $35 \times 10 \mathrm{~mm}$ tissue culture dish (Corning, catalog number: 353001)

10. SecureSeal ${ }^{\mathrm{TM}}$ imaging spacer (Grace Bio-Labs, catalog number: 654006)

11. $22 \times 22 \mathrm{~mm}$ (No. 1.5) micro coverslip (VWR, catalog number: 48366-227)

12. Adult (> P21) male and female C57BL/6J mice (JAX, catalog number: 000664)

13. Fluriso ${ }^{\mathrm{TM}}$ Isofluorane, USP (VetOne, catalog number: NDC 13985-528-60)

14. Ketamine (or similar as approved by your local regulations)

15. Xylazine (or similar as approved by your local regulations)

16. Buprenorphine $\mathrm{HCl}$ (or similar as approved by your local regulations)

17. Refresh Tears ${ }^{\circledR}$ Lubricant Eye Drops (Allergan, catalog number: NDC 0023-0798-01, or similar)

18. Proparacaine Hydrochloride Ophthalmic Solution, USP (Sandoz, catalog number: NDC 61314016-01)

19. Cholera Toxin Subunit B (CTB), Alexa Fluor ${ }^{\mathrm{TM}} 555$ Conjugate (Thermo Fisher, catalog number: C34776)

20. 10x Phosphate Buffered Saline (Thermo Fisher, catalog number: AM9624)

21. Paraformaldehyde 16\% Solution (Fisher Scientific, catalog number: 50-980-488) 
22. FocusClear ${ }^{\mathrm{TM}}$ (CelExplorer, catalog number: FC-101)

23. MountClear ${ }^{\mathrm{TM}}$ (CelExplorer, catalog number: MC-301)

24. Triton $^{\mathrm{TM}} \mathrm{X}-100$ (Sigma, catalog number: $\mathrm{X} 100$ )

25. Non-specific Goat serum (Thermo Fisher, catalog number: 16-210-064)

26. Anti-RBPMS, Guinea Pig (Phospho Solutions, catalog number: 1832-RBPMS)

27. Anti-Guinea Pig-Alexa Fluor 647 (Thermo Fisher, catalog number: A21450)

28. ProLong ${ }^{\circledR}$ Gold Antifade Mountant (Thermo Fisher, catalog number: P36934)

29. Flat mount blocking buffer (see Recipes)

\section{Equipment}

1. Surgical Microscope (WPI, catalog number: PSMB5N)

2. Laboratory Stereo Microscope (VWR, catalog number: 10836-004)

3. Hot Bead Sterilizer (FST, catalog number: 1800050)

4. Mouse Heating Pad (Stryker, catalog number: TP600/700)

5. Tabletop Isoflurane Anesthesia System with Induction Box (Harvard Apparatus, catalog number: 72-6468)

6. Dumont \#5 Straight Forceps (FST, catalog number: 11251-10)

7. Dumont \#7 Curved Forceps (FST, catalog number: 11271-30)

8. Dumont \#N5 Forceps Cross Action Inox Thin Tips Size .05 x .01 Biologie Tips (Roboz Surgical, catalog number: RS-5020)

9. Hamilton Syringe (Hamilton Company, $5 \mu \mathrm{l}, 700$ series, catalog number: 7634-01)

10. Hamilton 33 gauge needle (Hamilton Company, catalog number: 7803-05, 33 gauge, point 4 , 0.375 inches)

11. Vannas Spring Scissors-2.5 mm Cutting Edge (FST, catalog number: 15000-08)

12. Surgical Scissors (FST, catalog number: 91402-14)

13. Zeiss 880 LSM Confocal Microscope (Zeiss)

\section{Software}

1. Fiji ImageJ (https://imagej.net/Fiii)

2. Adobe Photoshop (https://www.adobe.com/)

\section{Procedure}

A. Intravitreal AAV2 injection

1. Anesthetize mice in accordance with your lab's and your Institutional Animal Care and Use Committee (IACUC)-approved protocols or country-specific regulations. For the purpose of 
intravitreal injections, isoflurane administered using a tabletop induction box and nose cone is sufficient.

2. While animals are under anesthesia, make sure to keep their eyes moist at all times by applying Refresh Tears ${ }^{\circledR}$ lubricating eye drops as needed (one drop per eye every 5 min is sufficient).

Note: To prevent the cornea from drying out during intravitreal injections and ONC surgeries liberally apply Refresh Tears ${ }^{\circledR}$ lubricating eye drops as required.

3. Once anesthetized, transfer a mouse to the surgical scope and attach nose cone (make sure isoflurane is flowing).

4. Instill 1 drop of proparacaine (or similar topical anesthetic) to each eye to block local reflexes. Wait $~ 1-2$ min and confirm the depth of anesthesia by gently touching the conjunctiva.

5. Tilt the mouse's head such that the targeted eye is facing the scope (Figure 1A). Use a cotton tipped applicator to wipe away any excess liquid.

6. Using \#5 forceps, slide the tips between the upper and lower eyelids such that the eyeball protrudes out of the socket and lightly pinch the conjunctiva $\sim 1 \mathrm{~mm}$ below the limbus (Figure 1B).

7. While holding the eyeball steady with forceps, pick up a preloaded $5 \mu$ Hamilton syringe with your free hand and at a $60^{\circ}$ angle with respect to the optic nerve, carefully insert the needle $\sim 1 \mathrm{~mm}$ (bevel facing towards the lens) through the sclera $\sim 1 \mathrm{~mm}$ below the limbus into the vitreous cavity (Figure 1C). Since the lens occupies the majority of the vitreous cavity, care should be taken to avoid piercing the lens.

Note: Purified in vivo grade AAV2 with a titer $\geq 1 \times 10^{12} \mathrm{GC} / \mathrm{ml}$ should be used to efficiently transduce RGCs.

8. Using your index finger, gently push on the plunger to inject $1 \mu \mathrm{l}$ of virus.

Note: Check the lens before optic nerve crush. If there is cataract, the eye should be excluded.

9. After injecting, leave the needle in the vitreous cavity for $\sim 15-20 \mathrm{sec}$ to prevent leakage, then slowly remove.

Note: If liquid starts to flow back out of the site of injection you may have injected too much virus and the eye may be excluded from future analysis.

10. Remove forceps and gently place the eye back into the socket by closing the eyelids.

11. Apply a generous amount of Polycin ${ }^{\circledR}$ Ophthalmic Ointment (or similar antibiotic ointment) over the cornea and injection area.

12. Flip mouse and repeat the injection in the contralateral eye if desired.

13. Place animals on a heating pad to fully recover $(\sim 2-3 \mathrm{~min})$ before transferring back to their home cages.

14. House animals for 2 weeks, or optimized length, to allow for maximum virus expression then proceed to the optic nerve crush. 

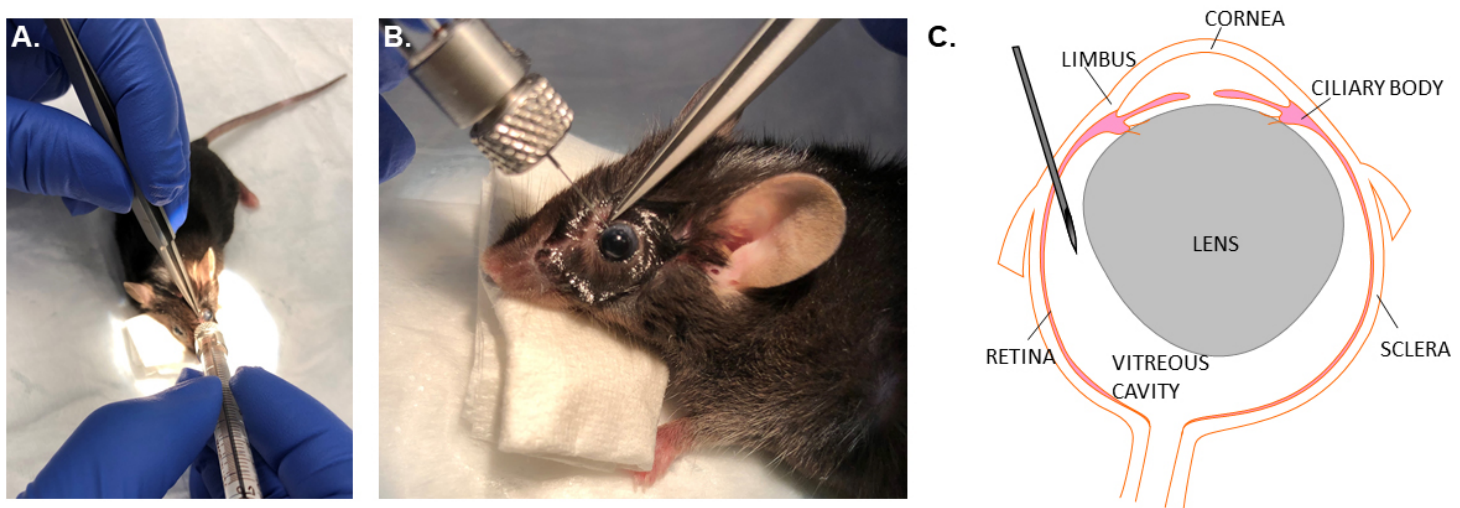

Figure 1. Intravitreal injection of AAV2. A. Transfer an anesthetized mouse to the surgical scope and tilt the head such that the targeted eye is facing the scope. Instill one drop of proparacaine on each eye to block local reflexes and clean away any excess liquid with a cotton tipped applicator. B. Pinch the conjunctiva just below the limbus with forceps and hold the eye steady. C. With your free hand, insert preloaded Hamilton syringe at a $60^{\circ}$ angle just below the limbus $\sim 1 \mathrm{~mm}$ into the vitreous cavity and gently inject $1 \mu \mathrm{l}$ of virus by depressing the plunger with your index finger. Hold needle in place for $\sim 15-20 \mathrm{~s}$ before removing to prevent leakage and to allow virus to distribute evenly.

B. Optic nerve crush surgery

1. Deeply anesthetize mice by isoflurane induction and intraperitoneal (IP) or subcutaneous (SC) injection of ketamine (100 mg/kg)/xylazine $(20 \mathrm{mg} / \mathrm{kg}$ ) (or similar approved cocktail) in accordance with your lab's and institutional IACUC protocols or local regulations

Note: To prevent the cornea from drying out during intravitreal injections and ONC surgeries liberally apply Refresh Tears ${ }^{\circledR}$ lubricating eye drops as required.

2. Transfer a mouse to the surgical scope and instill 1 drop of proparacaine (or similar topical anesthetic) to each eye. Wait $\sim 1-2$ min and confirm the depth of anesthesia by hindlimb pinch.

3. After ensuring the depth of anesthesia, tilt the mouse's head a little such that the targeted eye is facing the surgeon.

4. Brush any hair and excess liquid away from the eye with a wet cotton tipped applicator.

5. Before starting the surgical procedure, surgical tools should be sterilized using a hot bead sterilizer. Wait a few seconds after sterilization to allow tools to cold down.

6. Slide the tips of $\# 5$ forceps between the upper and lower eyelids such that the eye protrudes out of the socket.

7. With the opposite hand pinch the conjunctiva along the superior-temporal border of the globe with \#7 curved forceps making a small fold in the tissue (this will prevent cutting too deep and damaging the sclera) (Figure 2A).

8. Retract \#5 forceps and pick up spring scissors maintaining your hold of the eyeball with curved forceps.

9. With spring scissors, cut a $1 \mathrm{~mm}$ hole in the superior-temporal conjunctiva using the fold as a 
Please cite this article as: Cameron et. al., (2020). Optic Nerve Crush in Mice to Study Retinal Ganglion Cell Survival and Regeneration,Bio-protocol 10

guide (Figure 2A) and then blunt dissect the conjunctiva to make the hole bigger (Figure 2B).

10. Gently separate the tissue with forceps till you have a clear view of the superior muscle (this can be challenging to identify but is important to prevent damage of the orbital sinus) (Figures 2C-2D).

11. With your dominant hand, insert \#5 forceps through the newly formed opening past the superior muscle and carefully push away any fat that is obstructing the optic nerve (Figure 2E). Take care not to disrupt any blood vessels, particularly the orbital sinus, or the cavity will fill with blood and completely obstruct your view of the optic nerve.

Note: There can be a little bit of bleeding during the tissue separation; if so, use cotton tips to wipe away blood.

12. Once the optic nerve is visualized and cleared, insert the Cross Action self-closing forceps in the open position around the optic nerve $2 \mathrm{~mm}$ behind the eyeball, loosen grip and hold steady for 3 secs to crush (Figure 2F). This should be adequate to induce complete degeneration of optic nerve axons up to the crush site and significant RGC cell death.

Note: The central retinal artery (CRA) of mice branches off the ophthalmic artery (OA), where is very close to the globe (May and Lütjen-Drecoll, 2002). If you clear the tissue around the ON and crush $2 \mathrm{~mm}$ behind the globe, the crush won't obstruct the blood supply of retina.

13. Following crush, open and retract Cross Action self-closing forceps.

14. Gently coax the eye back to its natural position with forceps and close the eyelids.

15. Apply a generous amount of Polycin ${ }^{\circledR}$ Ophthalmic Ointment (or similar antibiotic ointment) over the incision and cornea.

16. Turn the mouse's head and repeat crush on the contralateral optic nerve experimental plan.

17. Administer Buprenorphine $\mathrm{HCl}$ (or similar analgesic) after surgery in accordance with your lab's and institutional IACUC protocols or local regulations.

18. Place mice on a heating pad to fully recover ( $\sim 60 \mathrm{~min})$ before transferring back to their home cages.

19. Check for infection every $12-24 \mathrm{~h}$ as dictated by your lab's and institutional IACUC protocols or local regulations.

20. House mice for 12 days then proceed to the CTB injections. 


\section{bio-protocol
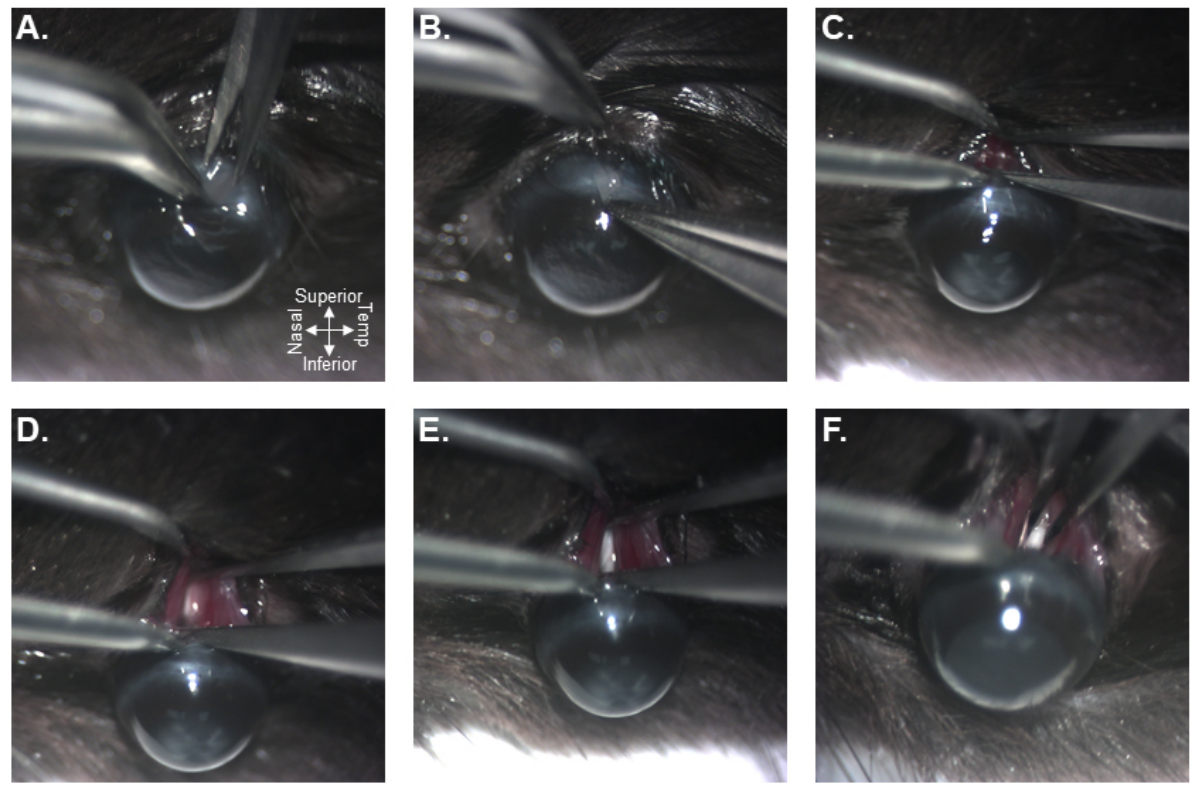

Figure 2. Optic nerve crush surgery. A. Pinch the conjunctiva along the superior-temporal border of the globe with forceps making a small fold in the tissue. Using spring scissors incise a $1 \mathrm{~mm}$ hole cutting posteriorly using the folded conjunctiva as a guide. B. Bluntly but gently separate the tissue with forceps to enlarge the hole $\sim 2-3 \mathrm{~mm}$. C-D. Carefully push away any fat obstructing the optic nerve taking care not to disrupt the orbital sinus. E. Once the optic nerve is cleared and visualized. F. use Cross Action self-closing forceps to crush the optic nerve $2 \mathrm{~mm}$ behind the globe for $3 \mathrm{~s}$.

C. CTB labeling of regenerating retinal ganglion cell axons

1. Resuspend CTB-555 (or desired fluorophore, $500 \mu \mathrm{g}$ ) in $50 \mu \mathrm{l}$ of sterile PBS to a final concentration of $10 \mu \mathrm{g} / \mu \mathrm{l}$.

2. 12 days post-crush, anesthetize mice accordingly and inject $1 \mu \mathrm{l}$ of CTB-555 into each eye intravitreally as per the AAV2 injection protocol described above.

Notes:

a. To prevent the cornea from drying out during intravitreal injections and ONC surgeries liberally apply Refresh Tears ${ }^{\circledR}$ lubricating eye drops as required.

b. CTB-555 should be used with AAV2-GFP viral vectors. Alternatively, CTB-488 or -647 can be used depending on the AAV2 reporter.

c. Regenerating neurons can alternatively be visualized by GAP43 staining as described in Leon et al. (2000).

D. Tissue dissection and optic nerve clearing

1. Sacrifice mice 14 days post-crush by transcardial perfusion in accordance with your lab's and institutional IACUC protocols or local regulations.

2. Decapitate fixed mice and remove all the skin and hair around the eyes and top of the head with surgical scissors (Figure 3A). 
3. Using \#5 forceps and spring scissors, carefully dissect away the conjunctiva connecting the globe to the socket such that it can freely move within the orbit.

4. Gently pull the globe nasally to access the orbital cavity and remove the lacrimal glands as well as any fat from behind the eye to expose the optic nerve.

5. Enucleate eyes by cutting the optic nerve as close to the globe as possible with spring scissors and transfer to 48 -well dish containing $\sim 500 \mu \mathrm{l} 4 \%$ PFA. Post-fix for $1 \mathrm{~h}$ at room temperature (Figures 3A-3B).

6. After $1 \mathrm{~h}$, aspirate the PFA and replace with $1 \times$ PBS. Eyes can be stored in sterile $1 \mathrm{x}$ PBS for $12+$ hours at $4{ }^{\circ} \mathrm{C}$.

7. Once all the eyes have been post-fixed proceed to Flat mount dissection.

8. To isolate optic nerves, first cut away the hindbrain with large surgical scissors.

9. Then carefully cut along the sagittal (dorsal) and squamous (temporal) sutures with surgical scissors and peel the skull nasally to expose the cortex (Figures 3B-3C).

10. Bluntly separate the olfactory bulbs from the cortex with $\# 5$ forceps, then slide forceps under the forebrain, and gently lift $\sim 45^{\circ}$ from front to back to expose the optic nerves at the base of the skull (Figure 3D).

11. Make sure the nerves can freely slide through the meningeal sheath and flip the brain out of the skull (Figure 3E).

Note: If the nerves get stuck dissect away the meninges before removing the brain.

12. With the underside of the brain exposed, use spring scissors to cut the nerves just beyond the optic chiasm (Figures 3F-3G).

13. If desired, separate the right and left optic nerves at the chiasm using spring scissors.

14. Transfer nerves to 48-well dish containing $\sim 300 \mu \mathrm{l}$ 4\% PFA/well and post-fix for $1 \mathrm{~h}$ at room temperature.

15. After fixation, remove PFA with a transfer pipette and replace with $\sim 100 \mu \mathrm{l} \mathrm{FocusClear}{ }^{\mathrm{TM}}$ for $\geq 3$ $\mathrm{h}$ or until optic nerve is totally transparent (Diekmann et al., 2015; Wang et al., 2019)

16. Place a SecureSeal ${ }^{\mathrm{TM}}$ imaging spacer on a clean slide to build a small chamber to prevent squashing the nerve while mounting.

17. Place optic nerve in chamber, cover with MountClear ${ }^{\mathrm{TM}}$ and coverslip with a $22 \times 22 \mathrm{~mm}$ micro coverslip.

Note: Make sure to warm MountClear ${ }^{T M}$ in a $55{ }^{\circ} \mathrm{C}$ water bath for about 30 min prior to the mounting.

18. Image whole mounted cleared optic nerves from the crush site to the chiasm at 20x by tile scanning and Z-stacking on a confocal microscope. Stacked images should be acquired at $8 \mu \mathrm{m}$ intervals.

19. Maximum project z-stack images and proceed to Quantification of cleared optic nerve regeneration. 

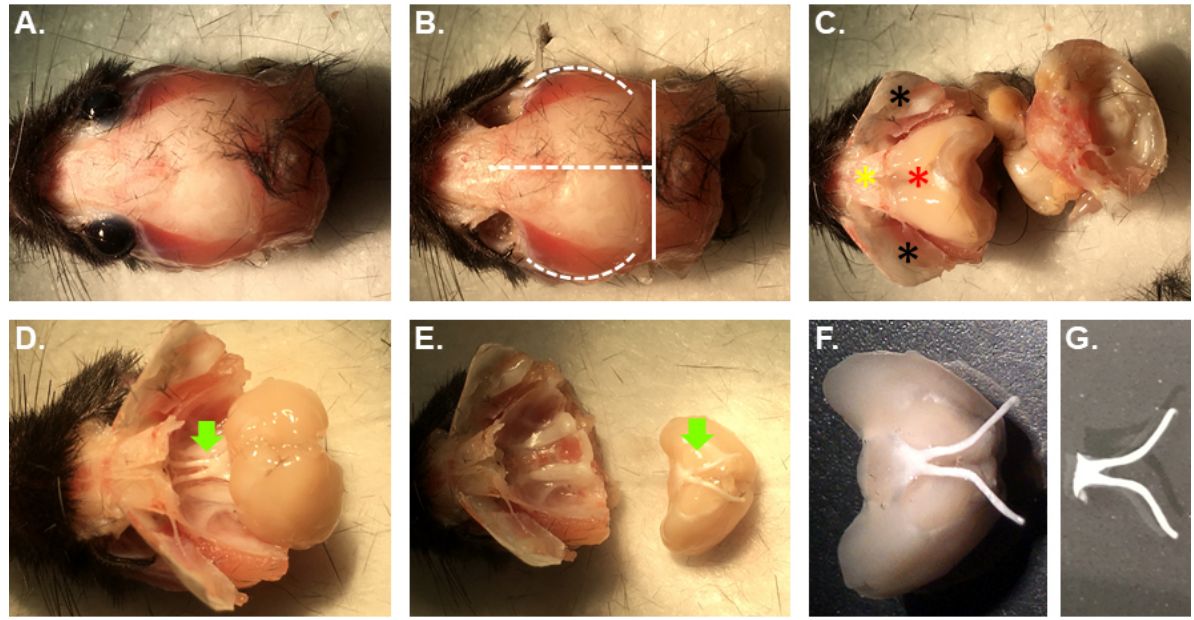

Figure 3. Eye and optic nerve dissection. A. Remove skin and hair around the eyes and top of the head with large surgical scissors, then using forceps and spring scissors carefully dissect away the conjunctiva connecting the globe to the socket and cut the optic nerve as it enters the globe. Transfer eyes to 48-well dish containing 4\% PFA. B. Using large surgical scissors cut away the hindbrain (solid line), then superficially cut along the sagittal and squamous sutures (dashed lines). C. Gently peel the skull (black asterisks) nasally to expose the cortex (red asterisk) and olfactory bulbs (yellow asterisk). D. Bluntly separate the olfactory bulbs from the cortex, then gently slide forceps under the forebrain and lift $\sim 45^{\circ}$ to expose the optic nerves (green arrow). E. To isolate nerves, carefully flip the brain out of the skull making sure the nerves can freely slide through the meningeal sheath before removal (if necessary, dissect away the meninges to prevent optic nerves from catching and tearing). F. With the underside of the brain exposed, G. cut the nerves just beyond the chiasm and transfer to a 48 -well dish containing $4 \%$ PFA.

E. Flat mount dissection

1. Under a dissection scope, transfer fixed eyes to $35 \times 10 \mathrm{~mm}$ tissue culture dish filled with sterile 1x PBS (Figure 4A).

2. Hold the eyeball steady by pinching the cornea with $\# 5$ forceps and make a small hole just above the limbus with a needle (Figure 4B).

3. Pinch the cornea with forceps and dissect away with spring scissors using the newly formed hole as an access point (Figure 4C).

4. Carefully remove the lens and any remaining vitreous from the surface of the retina using \#5 forceps taking care not to touch the retinal surface (Figure 4D).

5. Slide $\# 5$ forceps behind the retina into the subretinal space and hold the globe steady by pinching the sclera.

6. Slide closed spring scissors into the subretinal space and move them around the circumference of the eye to completely detach the retina from the RPE/sclera.

7. Once detached, use spring scissors to partition the retina into equally sized quadrants by making 4 evenly spaced 2-3 mm cuts towards the center of the retina (Figure $4 \mathrm{E}$ ). 
8. Gently slide retina out of the eye cup and transfer into a separate $35 \times 10 \mathrm{~mm}$ tissue culture dish filled with sterile 1x PBS (Figure 4F).

9. Inspect the surface of the retina and remove any remaining vitreous.

Note: It is critical that all the vitreous is removed from the surface of the retina otherwise it will interfere with antibody staining.

10. Transfer dissected retina to a 48-well tissue culture dish containing 1x PBS using a cut $3 \mathrm{ml}$ transfer pipette (Figures 4G-4H) and proceed to Flat mount immunostaining.
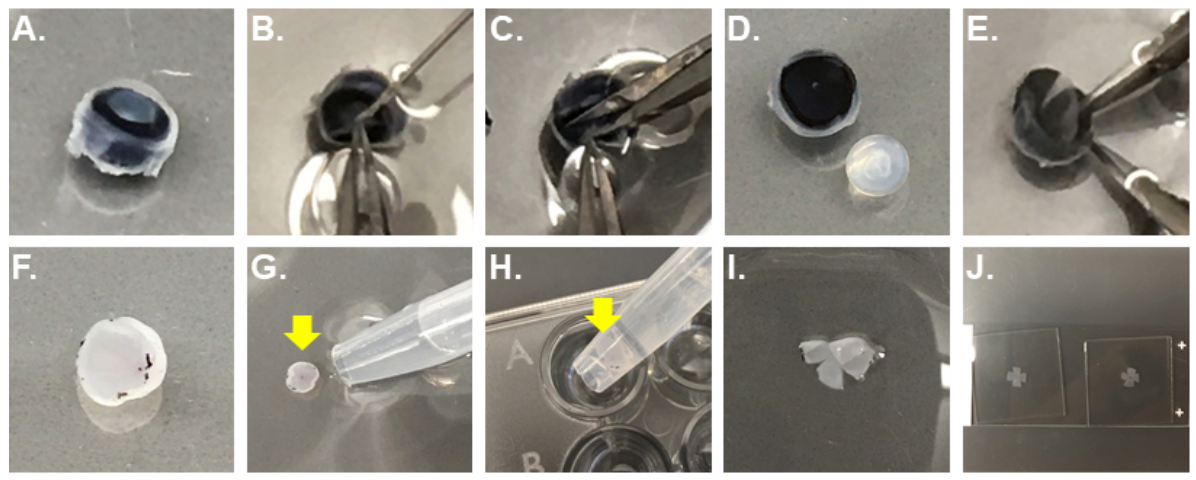

Figure 4. Retinal flat mount dissection and staining. A. Transfer fixed eyes to a small tissue culture dish containing fresh $1 x$ PBS. B. Hold eyeball steady with forceps and poke a small hole in the cornea with a needle just above the limbus. C. Using the newly formed hole as an access point, dissect away the cornea with spring scissors. Cut just below the limbus to ensure the ciliary body and iris are separated from the retina. D. Gently remove the lens and vitreous with forceps. E. Slide forceps into the subretinal space and use spring scissors to detach the retina from the RPE/sclera. Partition retina into quadrants by making 4 evenly spaced $\sim 2-3 \mathrm{~mm}$ cuts towards the optic nerve head. F. Carefully slide the retina out of the eye cup and remove any remaining vitreous on the retinal surface with forceps. G. Using a cut $3 \mathrm{ml}$ transfer pipette, $\mathrm{H}$. transfer dissected retina (yellow arrow) to a 48-well dish containing 1x PBS and proceed with retinal staining as described below. I. Following staining, transfer retinas ganglion cell side up to a clean slide and unfurl petals. Remove any excess liquid with a twisted KimWipe taking care not to touch the retina. J. Add 1-2 drops ProLong ${ }^{\circledR}$ Gold Antifade Mountant on the surface of each retina and coverslip. Let dry overnight on a flat surface before imaging.

F. Flat mount immunostaining

1. Aspirate PBS from each well and replace with $500 \mu \mathrm{l}$ of Flat Mount Blocking Buffer. Incubate for 1-2 $\mathrm{h}$ at room temperature on a rocker.

2. While blocking, dilute the primary antibody (Guinea Pig-Anti-RBPMS) 1:250 in Flat Mount Blocking Buffer.

3. After $1-2 \mathrm{~h}$, aspirate the blocking buffer and add $\sim 200 \mu \mathrm{l}$ of the diluted primary antibody to each well. Incubate for $24-48 \mathrm{~h}$ at $4{ }^{\circ} \mathrm{C}$ on a rocker.

4. Aspirate primary antibody and wash retinas 3 times with $1 \times$ PBS for 15 min each. 
5. Dilute the secondary antibody (Anti-Guinea Pig-Alexa Fluor 647) 1:500 in Flat Mount Blocking Buffer.

6. Aspirate final wash and add $\sim 200 \mu$ of the diluted secondary to each well. Incubate for $2-4 \mathrm{~h}$ at room temperature on a rocker.

Note: Alternatively, retinas can be incubated for $24 \mathrm{~h}$ at $4{ }^{\circ} \mathrm{C}$ on a rocker.

7. Aspirate secondary antibody and wash retinas 3 times with $1 x$ PBS for 15 min each.

8. Following the final wash, transfer the retinas to a clean slide using a cut $3 \mathrm{ml}$ transfer pipette.

9. Place retinas ganglion cell side facing up and unfurl the petals such that they are as flat as possible (Figure 4I). Remove any excess PBS using a Kimwipe taking care to not touch the retina.

10. Place 1-2 drops of ProLong ${ }^{\circledR}$ Gold Antifade Mountant on the surface of each retina and coverslip with a $22 \times 22 \mathrm{~mm}$ micro coverslip (Figure 4J).

11. Allow slides to set overnight on a flat surface protected from light.

12. Image the retinas at $10 x$ on a confocal microscope acquiring a tile-scan of the whole flatmounted retinas Z-stacked through the RGC layer (Figures 5A-5B).

13. Max intensity project the Z-stacks and proceed to Quantification of RGC survival.

\section{Data analysis}

A. Quantification of RGC survival

1. Before counting, set scale for each exported TIFF images accordingly.

2. Manually count RGCs in the peripheral ( $\sim 150-200 \mu \mathrm{m}$ away from the edge of the retina), middle ( $700-800 \mu \mathrm{m}$ away from the edge) and central ( 1,500-1,600 $\mu \mathrm{m}$ away from the edge) regions of each quadrant using the "cell counter" function in FIJI ImageJ (Figure $5 \mathrm{C}$ ).

3. Calculate average RGC cell density (D) per region (central, middle, periphery) using the formula: $D=[(R 1+R 2+R 3+R 4) / 4] / A$ where $R$ equals the number of RGCs for a given image and $A$ equals area $\left(\mathrm{mm}^{2}\right)$.

4. Calculate whole retinal RGC density using the formula: $\left(D_{c}+D_{M}+D_{P}\right) / 3$ where $D_{c}, D_{M}$, and $D_{P}$ represent the average densities of the central, middle, and peripheral regions, respectively (Figure 5D). 
Please cite this article as: Cameron et. al., (2020). Optic Nerve Crush in Mice to Study Retinal Ganglion Cell Survival and Regeneration,Bio-protocol 10 (6): e3559. DOI: 10.21769/BioProtoc.3559.

\section{bio-protocol
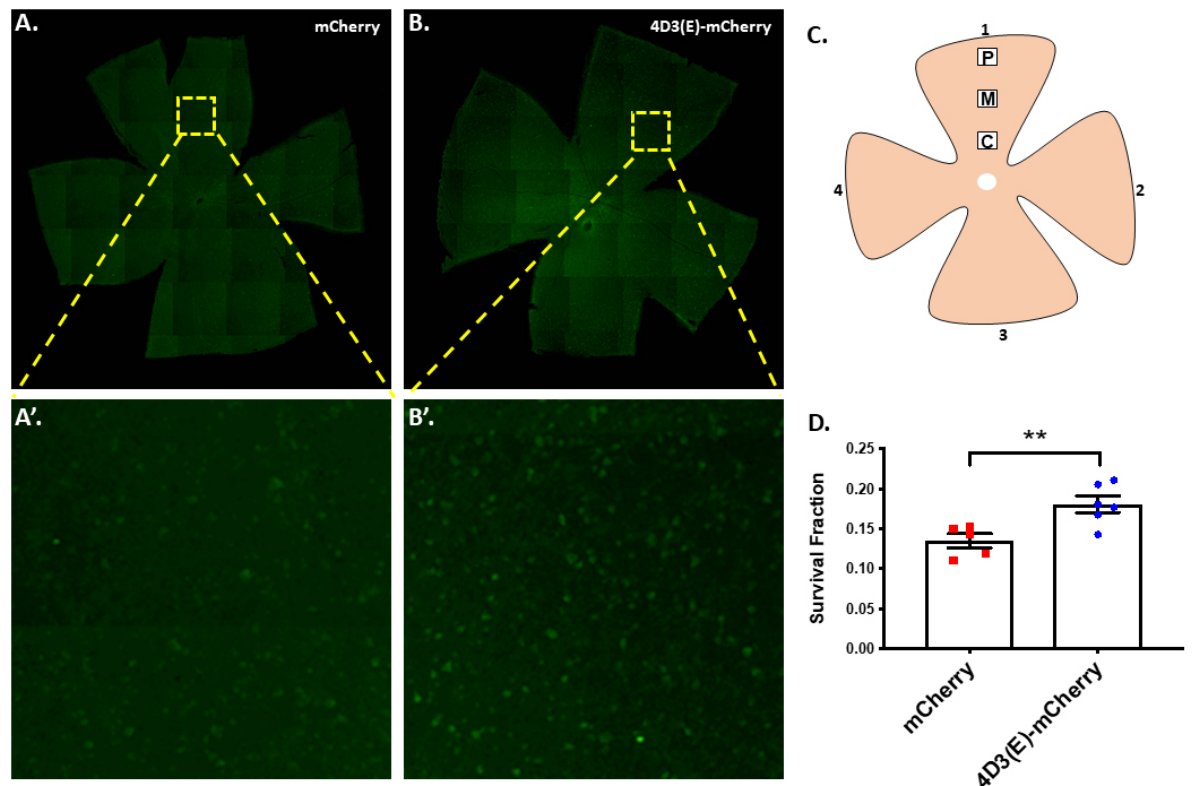

Figure 5. Quantification of RGC survival. 10x tile scanned/Z-projected confocal images of $A$. AAV2-mCherry and B. AAV2-4D3(E)-mCherry treated retinal flat mounts $C$. Count the number of RBPMS+ cells in the central $(C)$, middle $(M)$, and peripheral $(P)$ regions of each quadrant and calculate RGC density as describe above. D. Comparison of RGC survival of mCherry and 4D3(E) treated retinas adapted from Boczek et al., 2019.

B. Quantification of cleared optic nerve regeneration

1. Export and convert confocal images to TIFF format with $500 \mu \mathrm{m}$ scale bar.

2. Using Photoshop, make a metric scale ruler with red lines evenly spaced $250 \mu \mathrm{ms}$ apart and save as PSD format (Figure 6A).

3. Open both the optic nerve TIFF and PSD ruler images. Select the ruler and copy/paste to the optic nerve images. Use "Free Transform" under "Edit" to change the length of the ruler so that the distance between the first and third red lines equals $500 \mu \mathrm{m}$ (Figure 6B). Apply 2-3 rulers if the optic nerve is not straight (Figure 6C).

4. Manually count the number of CTB positive puncta that cross each red line starting $0.5 \mathrm{~mm}$ from the crush site and continuing all the way to the chiasm or to the end of longest regenerating axons. 
Please cite this article as: Cameron et. al., (2020). Optic Nerve Crush in Mice to Study Retinal Ganglion Cell Survival and Regeneration,Bio-protocol 10 (6): e3559. DOI: 10.21769/BioProtoc.3559.

\section{bio-protocol
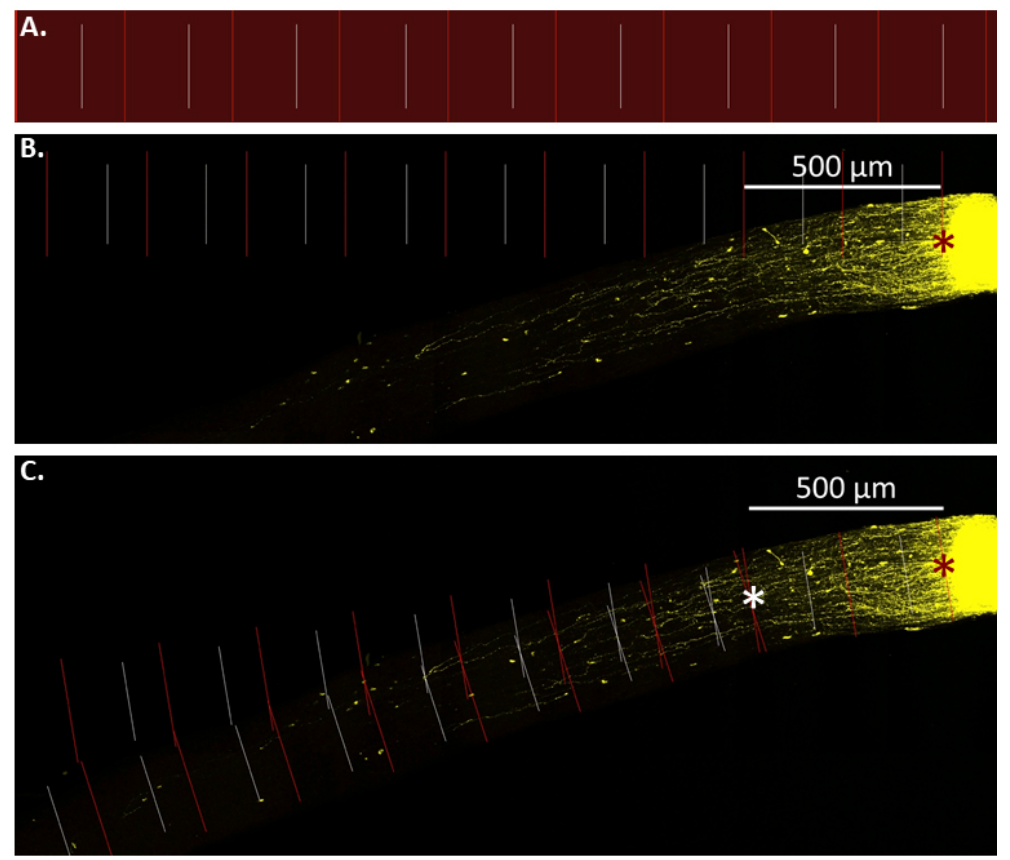

Figure 6. Quantification of regenerating axons. A. Create a ruler in Photoshop where the distance between two red lines is equal to $250 \mu \mathrm{m}$. B. Copy ruler to image layer and choose Free Transform to set the scale such that the distance between the first and third red line is $500 \mu \mathrm{m}$. C. Rotate the ruler and place the first red line over the crush site (red asterisks). If the optic nerve is not straight, copy another ruler and rotate according to the actual shape of optic nerve. White asterisk shows the connection of the first and second ruler.

\section{$\underline{\text { Recipes }}$}

1. Flat Mount Blocking Buffer

$1.2 \mathrm{ml}(3 \%)$ Triton X-100

$0.2 \mathrm{ml}(0.5 \%)$ Tween-20

$0.4 \mathrm{~g}(1 \%) \mathrm{BSA}$

$1 \mathrm{ml}(0.1 \%) 4 \%$ sodium azide

Fill to $40 \mathrm{ml} 2 \times$ PBS

\section{Acknowledgments}

This work was supported in part by National Institutes of Health Grants EY026766 (M.S.K. and J.L.G.), EY031167 (M.S.K.), P30-EY026877 to Stanford University, EY025915 (E.G.C.), and an unrestricted grant from Research to Prevent Blindness, Inc.

E.G.C., X.X., J.G., and M.A. participated in the design, data acquisition, and writing of the manuscript. J.L.G. and M.S.K. funded and supervised the work. All authors read and edited the manuscript. This protocol was adapted from Boczek et al. (2019) and Apara et al. (2017). 


\section{Competing interests}

The authors declare no competing interests.

\section{Ethics}

All animal experiments were approved and conducted in accordance with the guidelines of the Administrative Panel on Laboratory Animal Care (Protocol \#: 30550, exp.06/19/2020) at Stanford University and in compliance with the ARVO Statement for the Use of Animals in Ophthalmic and Vision Research.

\section{References}

1. Apara, A., Galvao, J., Wang, Y., Blackmore, M., Trillo, A., Iwao, K., Brown, D. P., Jr., Fernandes, K. A., Huang, A., Nguyen, T., Ashouri, M., Zhang, X., Shaw, P. X., Kunzevitzky, N. J., Moore, D. L., Libby, R. T. and Goldberg, J. L. (2017). KLF9 and JNK3 interact to suppress axon regeneration in the adult CNS. $J$ Neurosci 37(40): 9632-9644.

2. Boczek, T., Cameron, E. G., Yu, W., Xia, X., Shah, S. H., Castillo Chabeco, B., Galvao, J., Nahmou, M., Li, J., Thakur, H., Goldberg, J. L. and Kapiloff, M. S. (2019). Regulation of Neuronal Survival and Axon Growth by a Perinuclear cAMP Compartment. J Neurosci 39(28): 5466-5480.

3. Chang, E. E. and Goldberg, J. L. (2012). Glaucoma 2.0: neuroprotection, neuroregeneration, neuroenhancement. Ophthalmology 119(5): 979-986.

4. Diekmann, H., Kalbhen, P. and Fischer, D. (2015). Characterization of optic nerve regeneration using transgenic zebrafish. Front Cell Neurosci 9: 118.

5. Galvao, J., Iwao, K., Apara, A., Wang, Y., Ashouri, M., Shah, T. N., Blackmore, M., Kunzevitzky, N. J., Moore, D. L. and Goldberg, J. L. (2018). The Krüppel-like Factor Gene Target Dusp14 Regulates Axon Growth and Regeneration. Invest Ophthalmol Vis Sci 59 (7): 2736-2747.

6. Ito, Y. A., Belforte, N., Cueva Vargas, J. L. and Di Polo, A. (2016). A Magnetic Microbead Occlusion Model to Induce Ocular Hypertension-Dependent Glaucoma in Mice. J Vis Exp(109): e53731.

7. Templeton, J. P. and Geisert, E. E. (2012). A practical approach to optic nerve crush in the mouse. Mol Vis 18: 2147-2152.

8. Leon, S., Yin, Y., Nguyen, J., Irwin, N. and Benowitz, L. I. (2000). Lens injury stimulates axon regeneration in the mature rat optic nerve. $J$ Neurosci 20(12): 4615-4626.

9. Martin, K. R., Klein, R. L. and Quigley, H. A. (2002). Gene delivery to the eye using adenoassociated viral vectors. Methods 28(2): 267-275.

10. May, C. A. and Lütjen-Drecoll, E. (2002). Morphology of the murine optic nerve. Invest Ophthalmol Vis Sci 43(7): 2206-2212. 
Please cite this article as: Cameron et. al., (2020). Optic Nerve Crush in Mice to Study Retinal Ganglion Cell Survival and Regeneration,Bio-protocol 10

11. Moore, D. L., Blackmore, M. G., Hu, Y., Kaestner, K. H., Bixby, J. L., Lemmon, V. P. and Goldberg, J. L. (2009). KLF family members regulate intrinsic axon regeneration ability. Science 326(5950): 298-301.

12. Nickells, R. W., Schmitt, H. M., Maes, M. E. and Schlamp, C. L. (2017). AAV2-mediated transduction of the mouse retina after optic nerve injury. Invest Ophthalmol Vis Sci 58(14): 60916104.

13. Quigley, H. A. (2011). Glaucoma. Lancet 377(9774): 1367-1377.

14. Sappington, R. M., Carlson, B. J., Crish, S. D. and Calkins., D. J. (2010). The microbead occlusion model: A paradigm for induced ocular hypertension in rats and mice. Invest Ophthalmol Vis Sci 51 (1): 207-216.

15. Tang, Z., Zhang, S., Lee, C., Kumar, A., Arjunan, P., Li, Y., Zhang, F. and Li, X. (2011). An optiC nerve crush injury murine model to study retinal ganglion cell survival. $J$ Vis Exp (50).

16. Tham, Y. C., Li, X., Wong, T. Y., Quigley, H. A., Aung, T. and Cheng, C. Y. (2014). Global prevalence of glaucoma and projections of glaucoma burden through 2040: a systematic review and meta-analysis. Ophthalmology 121(11): 2081-2090.

17. Wang, J., Geisert, E. E. and Struebing, F. L. (2019). RNA sequencing profiling of the retina in C57BL/6J and DBA/2J mice: Enhancing the retinal microarray data sets from GeneNetwork. Mol Vis 25: 345-358.

18. Zhang, J., Li, L., Huang, H., Fang, F., Webber, H. C., Zhuang, P., Liu, L., Dalal, R., Tang, P. H., Mahajan, V. B., Sun, Y., Li, S., Zhang, M., Goldberg, J. L. and Hu, Y. (2019). Silicone oil-induced ocular hypertension and glaucomatous neurodegeneration in mouse. Elife 8: e45881. 